IJMMS 31:12 (2002) 761-763

PII. S0161171202111379

http://ijmms.hindawi.com

(c) Hindawi Publishing Corp.

\title{
A NEW TRIPLE SUM COMBINATORIAL IDENTITY
}

\section{JOSEPH SINYOR and AKALU TEFERA}

Received 10 November 2001 and in revised form 20 February 2002

We prove a new triple sum combinatorial identity derived from $r_{p}(x, y, z)=(x+y-z)^{p}$ $\left(x^{p}+y^{p}-z^{p}\right)$, extending a previous result by Sinyor et al.

2000 Mathematics Subject Classification: 05A19.

1. Introduction. The following combinatorial identity

$$
\begin{array}{r}
\sum_{i=0}^{n} \sum_{j=0}^{2 l} \sum_{k=0}^{j} \frac{(-1)^{i+j-k}}{m-l}\left(\begin{array}{c}
m+l-k \\
2 l-k+1
\end{array}\right)\left(\begin{array}{c}
m-l+k-1 \\
k
\end{array}\right)\left(\begin{array}{c}
2 l-j \\
n-i
\end{array}\right)\left(\begin{array}{l}
j \\
i
\end{array}\right) \\
= \begin{cases}\frac{1}{2 m+1}\left(\begin{array}{c}
2 m+1 \\
2 m-2 l+n
\end{array}\right)\left(\begin{array}{c}
m-l+n / 2 \\
n / 2
\end{array}\right), & \text { for } n \text { even, } \\
0, & \text { for } n \text { odd, }\end{cases}
\end{array}
$$

for $0 \leq l \leq m-1,0 \leq n \leq 2 l$, and $m, l, n$ nonnegative integers, arose from considering

$$
r_{p}(x, y, z)=(x+y-z)^{p}-\left(x^{p}+y^{p}-z^{p}\right),
$$

where $x, y, z$ are any nonzero integers, and $p$ is odd and $p>2$. We will use an identity proved in [2] which also arose from considering (1.2).

2. Proof of the identity. Define $f_{p}(x, y, z)=r_{p}(x, y, z) /(p(x+y)(z-x)(z-y))$. It is easily seen that $f_{p}(x, y, z)$ is a nonzero rational function and can be expressed as a polynomial in $(z-x)(z-y)$. Define coefficients $a_{i,(m-j)}$ such that

$$
f_{p}(x, y, z)=\sum_{j=0}^{m-1} \sum_{i=0}^{2 j} a_{i,(m-j)} x^{2 j-i} y^{i}(z-x)^{m-j-1}(z-y)^{m-j-1},
$$

where $p=2 m+1$. In [2] we proved the following combinatorial identity:

$$
\begin{gathered}
\sum_{l^{\prime} \leq l} \sum_{j^{\prime} \leq j} \frac{1}{m-l^{\prime}}\left(\begin{array}{c}
m+l^{\prime}-j^{\prime} \\
2 l^{\prime}-j^{\prime}+1
\end{array}\right)\left(\begin{array}{c}
m-l^{\prime}+j^{\prime}-1 \\
j^{\prime}
\end{array}\right)\left(\begin{array}{c}
m-l^{\prime} \\
2\left(l-l^{\prime}\right)-\left(j-j^{\prime}\right)
\end{array}\right)\left(\begin{array}{c}
m-l^{\prime} \\
j-j^{\prime}
\end{array}\right) \\
=\frac{1}{2(m-l)}\left(\begin{array}{c}
2 m \\
2 l+1
\end{array}\right)\left(\begin{array}{c}
2 l+1 \\
j
\end{array}\right)=\frac{1}{2 l+1}\left(\begin{array}{c}
2 m \\
2 l
\end{array}\right)\left(\begin{array}{c}
2 l+1 \\
j
\end{array}\right),
\end{gathered}
$$


and we showed that $a_{i,(m-j)}$ defined above satisfy a recurrence and are of the form

$$
a_{i,(m-j)}=\sum_{k=0}^{i} \frac{(-1)^{i-k}}{m-j}\left(\begin{array}{c}
m+j-k \\
2 j-k+1
\end{array}\right)\left(\begin{array}{c}
m-j+k-1 \\
k
\end{array}\right) .
$$

Now substitute (2.3) in (2.1) and change the variables $x$ and $y$ to $r=(x+y) / 2$, $s=(x-y) / 2$, which gives

$$
\begin{aligned}
& f_{p}(x, y, z)= \sum_{j=0}^{m-1} \sum_{i=0}^{2 j} a_{i,(m-j)}(r+s)^{2 j-i}(r-s)^{i}(z-x)^{m-j-1}(z-y)^{m-j-1} \\
&=\sum_{j=0}^{m-1} \sum_{i=0}^{2 j} \sum_{u=0}^{2 j} \sum_{v=0}^{u} \sum_{k=0}^{i} \frac{(-1)^{v+i-k}}{m-j}\left(\begin{array}{c}
m+j-k \\
2 j-k+1
\end{array}\right)\left(\begin{array}{c}
m-j+k-1 \\
k
\end{array}\right)\left(\begin{array}{c}
2 j-i \\
u-v
\end{array}\right)\left(\begin{array}{c}
i \\
v
\end{array}\right) \\
& \times r^{2 j-u} s^{u}(z-x)^{m-j-1}(z-y)^{m-j-1} .
\end{aligned}
$$

We now derive another form of $f_{p}(x, y, z)$ which will establish the identity.

Define $a=z-x$ and $b=z-y$. Then

$$
\begin{aligned}
r_{p}(x, y, z) & =(x+y-z)^{p}+z^{p}-x^{p}-y^{p} \\
& =\left(\frac{2 r-a-b}{2}\right)^{p}+\left(\frac{2 r+a+b}{2}\right)^{p}-\left(\frac{2 r-a+b}{2}\right)^{p}-\left(\frac{2 r+a-b}{2}\right)^{p} \\
& =r^{p}\left[\left(1-\frac{a+b}{2 r}\right)^{p}+\left(1+\frac{a+b}{2 r}\right)^{p}-\left(1+\frac{b-a}{2 r}\right)^{p}-\left(1-\frac{b-a}{2 r}\right)^{p}\right] .
\end{aligned}
$$

Using an identity from Gould [1], $\sum_{k=0}^{\lfloor n / 2\rfloor}\left(\begin{array}{c}n \\ 2 k\end{array}\right) x^{k}=\left((1+\sqrt{x})^{n}+(1-\sqrt{x})^{n}\right) / 2,(2.5)$ becomes

$$
\begin{aligned}
r_{p}(x, y, z) & =2 r^{p} \sum_{t=0}^{\lfloor p / 2\rfloor}\left(\begin{array}{c}
p \\
2 t
\end{array}\right)\left[\left(\frac{a+b}{2 r}\right)^{2 t}-\left(\frac{b-a}{2 r}\right)^{2 t}\right], \\
f_{p}(x, y, z) & =\frac{r^{p-1}}{p a b} \sum_{t=0}^{\lfloor p / 2\rfloor}\left(\begin{array}{c}
p \\
2 t
\end{array}\right)\left[\left(\frac{a+b}{2 r}\right)^{2 t}-\left(\frac{b-a}{2 r}\right)^{2 t}\right] \\
& =\sum_{t=0}^{\lfloor p / 2\rfloor} \frac{r^{2 m-2 t}}{p a b}\left(\begin{array}{c}
p \\
2 t
\end{array}\right)\left[\left(\frac{a+b}{2}\right)^{2 t}-\left(\frac{b-a}{2}\right)^{2 t}\right] \\
& =\sum_{t=0}^{\lfloor p / 2\rfloor} \frac{r^{2 m-2 t}}{p a b}\left(\begin{array}{c}
p \\
2 t
\end{array}\right)\left[\left(a b+s^{2}\right)^{t}-s^{2 t}\right] \\
& =\sum_{t} \frac{r^{2 m-2 t}}{p}\left(\begin{array}{c}
p \\
2 t
\end{array}\right) \sum_{i}\left(\begin{array}{l}
t \\
i
\end{array}\right) s^{2 i} a^{t-i-1} b^{t-i-1} .
\end{aligned}
$$


Substituting $j=m-t+i$, we obtain

$$
f_{p}(x, y, z)=\sum_{j} \sum_{i} \frac{1}{2 m+1}\left(\begin{array}{c}
2 m+1 \\
2 m-2 j+2 i
\end{array}\right)\left(\begin{array}{c}
m-j+i \\
i
\end{array}\right) r^{2 j-2 i} s^{2 i} a^{m-j-1} b^{m-j-1},
$$

where $0 \leq j \leq m-1$ and $0 \leq i \leq j$.

Note that (2.7) can be considered as an expression in $r^{2 j-i} s^{i}$ where all terms with odd $i$ are zero and $0 \leq i \leq 2 j$, that is,

$$
f_{p}(x, y, z)= \begin{cases}\sum_{j=0}^{m-1} \sum_{i=0}^{2 j} \frac{1}{2 m+1}\left(\begin{array}{c}
2 m+1 \\
2 m-2 j+i
\end{array}\right)\left(\begin{array}{c}
m-j+i / 2 \\
i / 2
\end{array}\right) r^{2 j-i} s^{i}(a b)^{m-j-1}, & i \text { even } \\
0, & i \text { odd } .\end{cases}
$$

We now equate (2.4) and (2.8), removing the common summation terms, which gives the identity.

REMARK 2.1. By using Wegschaider's MultiSum, a computer algebra package which is available from http://www.risc.uni-linz.ac.at/research/combinat/risc/software/, we found recurrence equations satisfied by the summand of our identity but we could not use them to give a short proof for our identity.

\section{REFERENCES}

[1] H. W. Gould, Combinatorial Identities. A Standardized Set of Tables Listing 500 Binomial Coefficient Summations, Henry W. Gould, West Virginia, 1972.

[2] J. Sinyor, T. Speevak, and A. Tefera, A new combinatorial identity, Int. J. Math. Math. Sci. 25 (2001), no. 6, 361-363.

Joseph Sinyor: Bell Nexxia, Floor 11 N, 483 Bay Street, Toronto, Ontario, Canada M5G 2E1

E-mail address: joseph.sinyor@be11nexxia.com

Akalu Tefera: Department of Mathematics, Grand Valley State University, ALLENDALE, MI 49401, USA

E-mail address: teferaa@gvsu.edu 


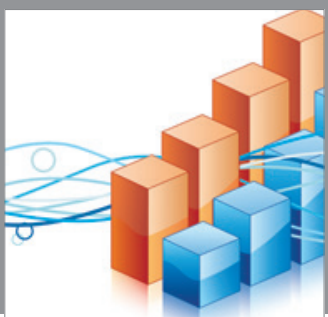

Advances in

Operations Research

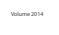

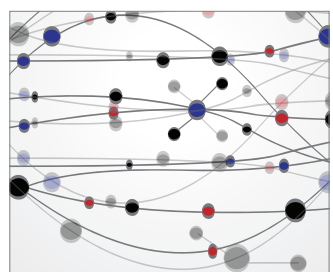

\section{The Scientific} World Journal
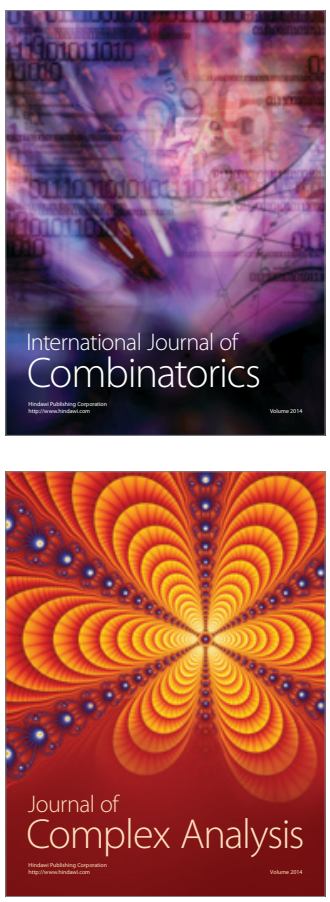

International Journal of

Mathematics and

Mathematical

Sciences
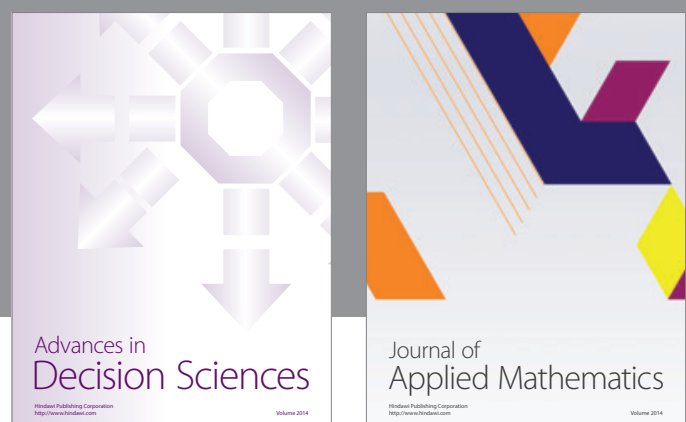

Journal of

Applied Mathematics
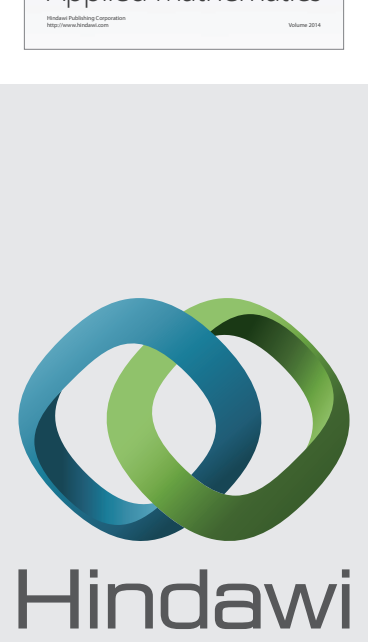

Submit your manuscripts at http://www.hindawi.com
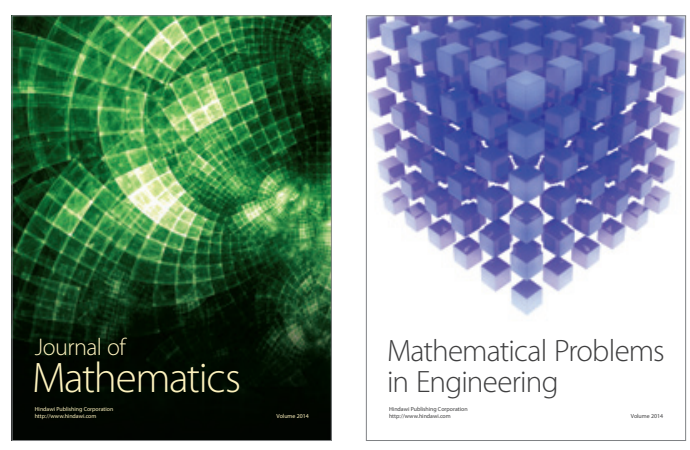

Mathematical Problems in Engineering
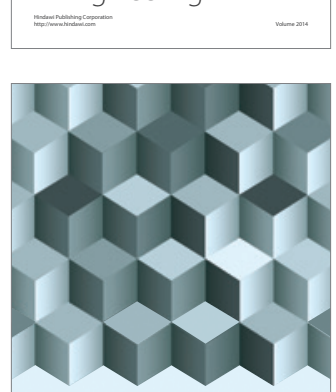

Journal of

Function Spaces
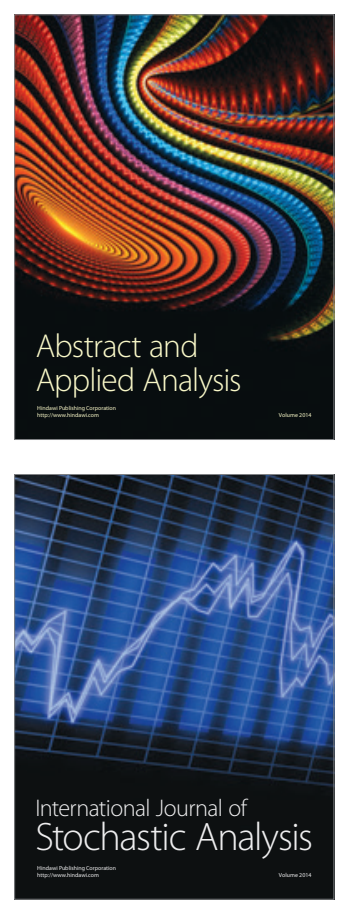

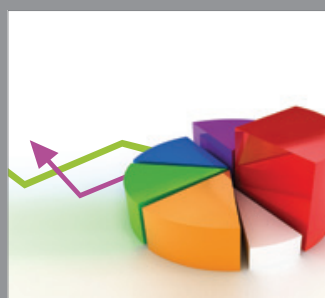

ournal of

Probability and Statistics

Promensencen
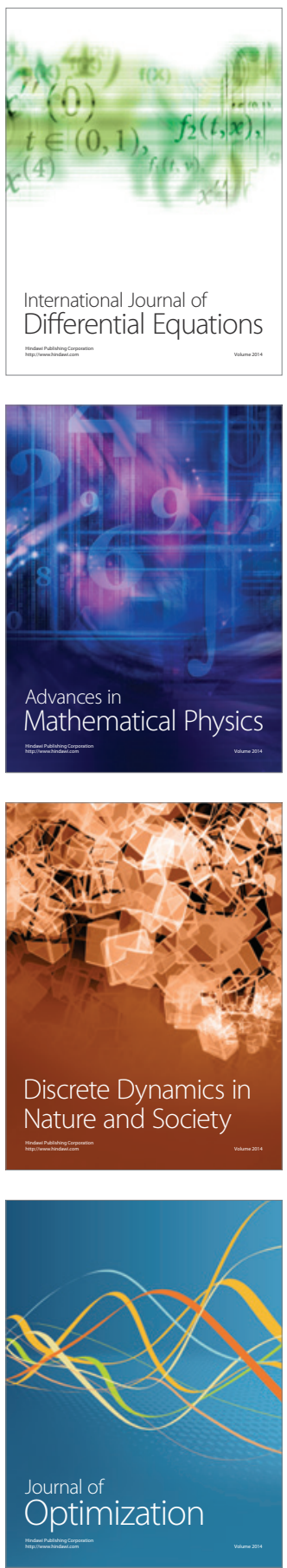